\title{
Low-Loss Single and Differential Semi-Coaxial Interconnects in Standard CMOS Process
}

\author{
Jun-De Jin ${ }^{1}$, Shawn S. H. Hsu ${ }^{1}$, Ming-Ta Yang ${ }^{2}$, and Sally Liu ${ }^{2}$ \\ ${ }^{1}$ Dept. of Electrical Engineering and Institute of Electronics Engineering, \\ National Tsing Hua University, Hsinchu, Taiwan 300, R.O.C. \\ ${ }^{2}$ Taiwan Semiconductor Manufacturing Company, Hsinchu, Taiwan 300-77, R.O.C. \\ Tel: 886-3-5731278; Fax: 886-3-5752120; Email: shhsu@ee.nthu.edu.tw
}

\begin{abstract}
The low-loss single semi-coaxial (S-SC) and differential semi-coaxial (D-SC) interconnects based on a standard 0.18- $\mu \mathrm{m}$ CMOS process are presented for the first time. Compared to the attenuation constant $(\alpha)$ reported for microstrip and CPW interconnects in CMOS process, the S-SC line shows the lowest loss of $0.90 \mathrm{~dB} / \mathrm{mm}$ at $50 \mathrm{GHz}$. The D-SC line also presents a very low differential-mode $\alpha$ of $\sim 1.00 \mathrm{~dB} / \mathrm{mm}$ at high frequencies. The characteristics of D-SC lines for differentialmode and common-mode are also investigated in details based on the measured mixed-mode S-parameters.
\end{abstract}

Index Terms - CMOS, CPW, differential line, microstrip, semi-coaxial interconnects.

\section{INTRODUCTION}

As the operation frequency of the silicon-based integratedcircuits dramatically increases, the impacts of interconnects on the circuit performance become non-negligible [1]-[2]. For high frequency circuits, the commonly used interconnect structures are the microstrip (MS) line [3]-[4] and coplanarwaveguide (CPW) [4]-[6]. For Si-based ICs, however, they may not be the best candidates due to the crosstalk between the signal lines (MS line) and the signal loss from the lossy $\mathrm{Si}$ substrate (CPW) [7].

Intuitively, a coaxial line is probably one of the best choices for low loss and low crosstalk interconnects at high frequencies. In this study, low-loss interconnects with a semicoaxial (SC) structure are realized by utilizing the multiple metal layers in modern CMOS process. Both single SC (S-SC) and differential SC (D-SC) lines are designed and characterized. With a semi-rounded ground plane, the SC line structure can be expected to present characteristics close to ideal transmission lines. Similar concepts have been reported in [8], but only with simulated results of S-SC lines in SOI process. Investigation on both $\mathrm{S}-\mathrm{SC}$ and $\mathrm{D}-\mathrm{SC}$ lines in standard CMOS technologies has not been reported to date.

In this paper, the low-loss S-SC and D-SC lines are designed and characterized from 0.2 to $50 \mathrm{GHz}$. Section II compares different interconnect structures, and describes the design procedure. Section III presents measured results and discussions of S-SC and D-SC lines. The characteristics of D$\mathrm{SC}$ lines for differential-mode and common-mode are also investigated in details based on the measured mixed-mode Sparameters [9]. Section IV concludes this work.

\section{DESIGN OF S-SC AND D-SC INTERCONNECTS}

Three different RF interconnect structures in CMOS process are shown in Fig. 1. As shown in Fig. 1(a), the MS line suffers from signal crosstalk, while possesses a good shielding from the lossy Si substrate. On the other hand, the CPW can be expected to show better crosstalk elimination by the two adjacent ground lines, as shown in Fig. 1(b). However, this structure cannot prevent the loss introduced by the unshielded $\mathrm{Si}$ substrate, which is referred as substrate skin effect [7]. By using a semi-rounded ground plane, as shown in Fig. 1(c) and (d), the SC lines provide a perfect shielding of the signal lines from the crosstalk and the lossy substrate to achieve a low-loss characteristic over a wide frequency range.

The designed SC lines were fabricated in a standard $1 \mathrm{P} 6 \mathrm{M}$ $0.18-\mu \mathrm{m}$ CMOS process. The signal line utilizes the top-metal layer (M6) with a thickness of $2.34 \mu \mathrm{m}$, while the semi-

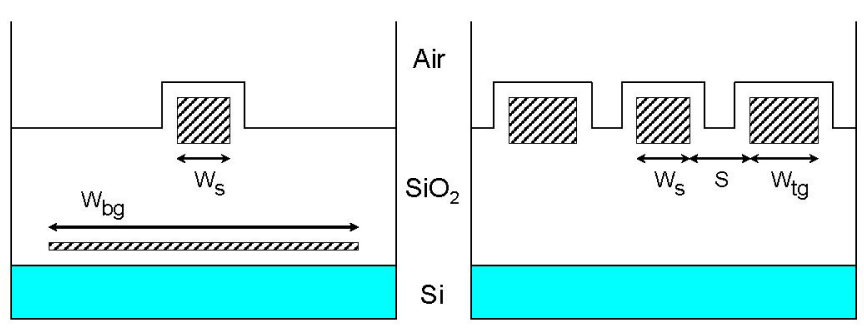

(a)

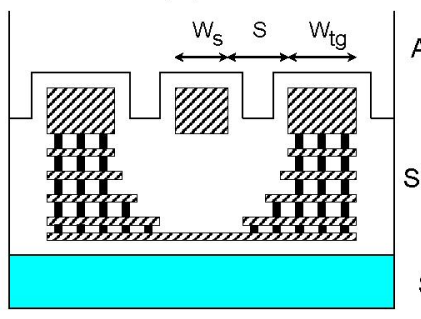

(c) (b)

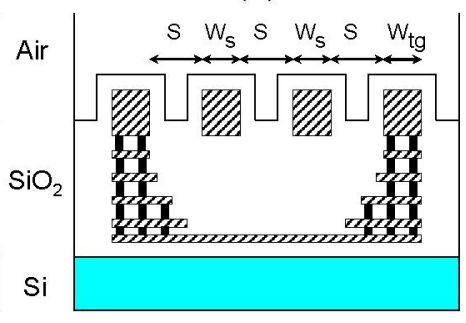

(d)
Fig. 1. RF interconnect structures for (a) MS line, (b) CPW, (c) SC line, (d) differential SC line in a standard 1P6M CMOS process. 
TABLE I

GEOMETRIES OF DESIGNED SC LINES $(\mathrm{L}=400 \mu \mathrm{m})$

\begin{tabular}{|c|c|c|c|c|}
\hline & $\begin{array}{c}\mathrm{W}_{\mathrm{s}} \\
(\mu \mathrm{m})\end{array}$ & $\begin{array}{c}\mathrm{S} \\
(\mu \mathrm{m})\end{array}$ & $\begin{array}{c}\mathrm{W}_{\mathrm{tg}} \\
(\mu \mathrm{m})\end{array}$ & $\begin{array}{c}Z_{0} \\
(\Omega)\end{array}$ \\
\hline S-SC1, D-SC1 & 15 & 2.4 & 15 & 33 \\
\hline S-SC2, D-SC2 & 15 & 79.5 & 15 & 50 \\
\hline S-SC3, D-SC3 & 5 & 2.4 & 5 & 50 \\
\hline S-SC4, D-SC4 & 5 & 10.8 & 5 & 75 \\
\hline
\end{tabular}

rounded ground planes employs the multiple metal layers from M1 to M6. For S-SC lines, the geometries were designed by the empirical equations for $Z_{0}[10]$ with three different characteristic impedances $\left(Z_{0}\right)$ of 33,50 , and $75 \Omega$, as summarized in Table $\mathrm{I}$. The $\mathrm{W}_{\mathrm{tg}}$ was designed the same as $\mathrm{W}_{\mathrm{s}}$, and the $\mathrm{W}_{\mathrm{s}}, \mathrm{S}$, and $\mathrm{W}_{\mathrm{tg}}$ of the D-SC lines are identical to those of the S-SC lines. The length of all SC lines is $400 \mu \mathrm{m}$ due to the limited chip area.

\section{MEASUREMENT RESULTS AND Discussions}

The S-SC lines were measured on-wafer with Cascade coplanar ground-signal-ground (GSG) infinity probes and Agilent 8510XF two-port vector network analyzer (VNA) from 0.2 to $50 \mathrm{GHz}$, and D-SC lines with GSGSG infinity probes and Agilent E8364A four-port PNA. For the Si-based interconnects measurements, de-embedding becomes a critical issue due to the low signal level and the lossy Si substrate. In this study, special cares were taken for the probing pads to achieve an accurate de-embedded procedure. First, a grounded M1 is applied underneath the probing pads to eliminate the generation of frequency-dependent series resistance and inductance from the lossy Si substrate [7]. Second, the pads area is minimized to $50 \times 50 \mu \mathrm{m}^{2}$ to alleviate the parallel parasitic capacitance.

\section{A. Characteristics of S-SC Lines}

After de-embedding, $Z_{0}$ and attenuation constant $(\alpha)$ of the $\mathrm{S}-\mathrm{SC}$ lines were both extracted from the measured $\mathrm{S}$ parameters [11], as shown in Fig. 2. The line S-SC2 presents an $\alpha$ of $0.90 \mathrm{~dB} / \mathrm{mm}$ at $50 \mathrm{GHz}$. Compared to the $\alpha$ values of $5.04 \mathrm{~dB} / \mathrm{mm}$ (MS line) and $1.22 \mathrm{~dB} / \mathrm{mm}$ (CPW) previously reported in a $0.13-\mu \mathrm{m}$ CMOS process with a top metal thickness of $3.3 \mu \mathrm{m} \mathrm{[4],} \mathrm{the} \mathrm{CMOS} \mathrm{SC} \mathrm{line} \mathrm{here} \mathrm{shows} \mathrm{a}$ substantially lower value even with a smaller top-metal thickness of $2.34 \mu \mathrm{m}$. This can be attributed to the lossy $\mathrm{Si}$ substrate is perfectly shielded and the semi-coaxial structure can support a wave propagation close to an ideal TEM mode.

Also observed from Fig. 2, the dependence of $\alpha$ on the interconnect geometry can be understood by the equation of a low-loss interconnect:

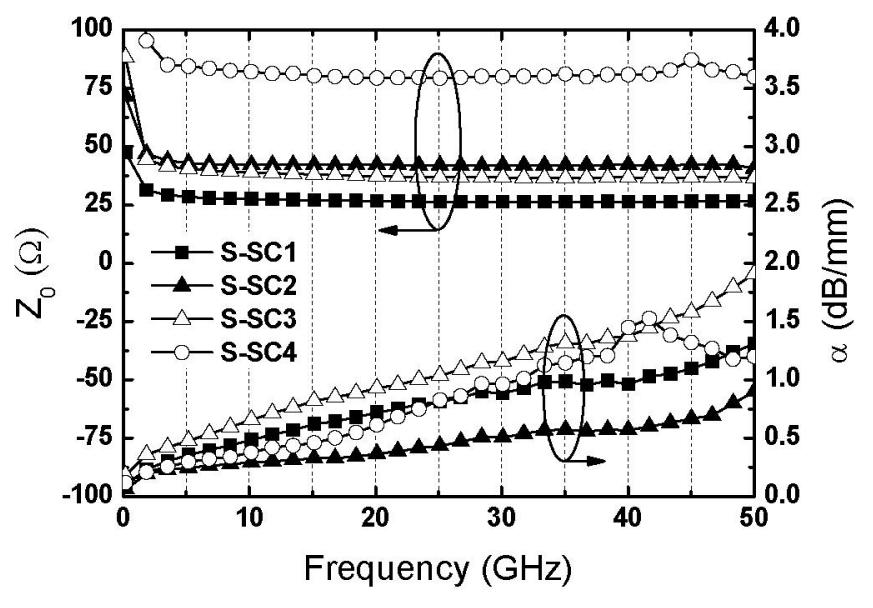

Fig. 2. $Z_{0}$ and $\alpha$ for $\mathrm{S}-\mathrm{SC}$ lines $(\mathrm{L}=400 \mu \mathrm{m})$.

$$
\alpha=\frac{R}{2 Z_{0}}
$$

where $R$ is the distributed resistance. As can be seen, $\alpha$ is not only proportional to $R$ but also inversely proportional to $Z_{0}$. From a comparison between the lines with the same $W_{\mathrm{s}}$, a larger $Z_{0}$ results in a smaller $\alpha$. For lines with the same $Z_{0}$, the wider line (S-SC2) presents a lower loss. The trends of $\alpha$ in Fig. 2 are all consistent with (1).

\section{B. Characteristics of D-SC Lines}

Since a four-port D-SC line is driven by both the differential- and common-mode signals, the RF characteristics are usually described using the mixed-mode S-parameters. Four $2 \times 2$ matrixes are included, and referred as the Sparameters of differential-mode $\left(S_{d d}\right)$, common-mode $\left(S_{c c}\right)$, differential-to-common-mode $\left(S_{d c}\right)$, and common-todifferential-mode $\left(S_{c d}\right)$. The measured four-port S-parameters of D-SC lines are converted to the mixed-mode S-parameters [12]. For a symmetric differential line as studied here, $S_{d c}$ and $S_{c d}$ are not existed, and only $S_{d d}$ and $S_{c c}$ are discussed. The measured differential-mode $Z_{0}\left(Z_{\text {odd }}\right)$ and $\alpha\left(\alpha_{d d}\right)$ can be calculated from $S_{d d}$, while the common-mode $Z_{0}\left(Z_{0 c c}\right)$ was obtained from $S_{c c}$. As shown in Fig. 3, the line D-SC2 shows the lowest $\alpha_{d d}$ of $\sim 1.00 \mathrm{~dB} / \mathrm{mm}$ at around $50 \mathrm{GHz}$, which can be explained by the similar reasons of the S-SC lines. In addition, for a weakly coupled differential line, $Z_{\text {odd }}$ should be $\sim 2 Z_{0}$ and $Z_{0 c c} \sim Z_{0} / 2$. This can be observed from line D-SC2 due to a large signal line spacing (S) of $79.5 \mu \mathrm{m}$ is employed.

Fig. 4(a) is the distributed $R L G C$ circuit model of a symmetric differential line including the coupling effect between the two signal lines. The distributed mutual inductance $\left(L_{m}\right)$ and capacitance $\left(C_{m}\right)$ describe the intensity of current coupling and voltage coupling, respectively. The fourport differential line model can be converted to one differential- and one common-mode two-port circuit models, 


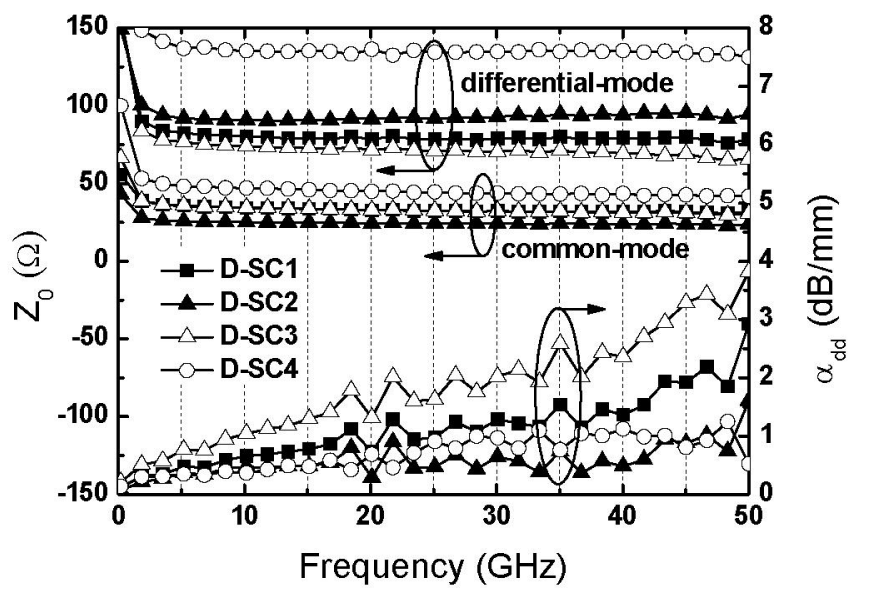

Fig. 3. Differential-mode $Z_{0}\left(Z_{\text {odd }}\right)$, common-mode $Z_{0}\left(Z_{0 c c}\right)$, and differential-mode $\alpha\left(\alpha_{d d}\right)$ for D-SC lines $(\mathrm{L}=400 \mu \mathrm{m})$.

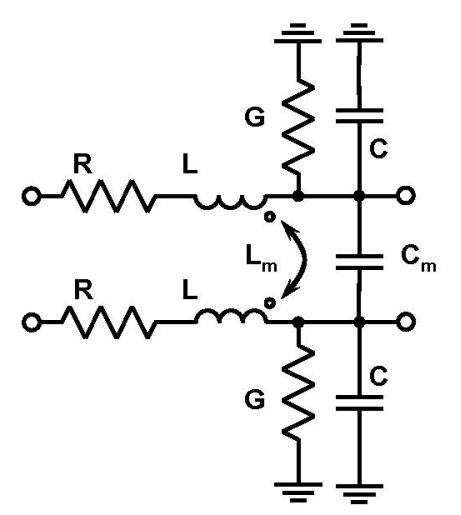

(a)

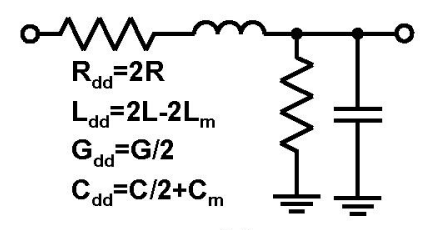

(b)

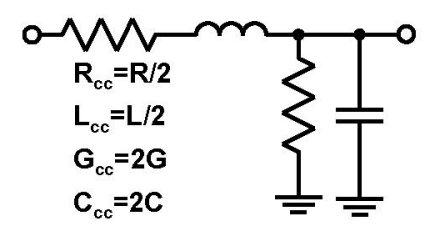

(c)
Fig. 4. Distributed $R L G C$ circuit model for a symmetric differential line: (a) four-port model, (b) differential-mode, (c) common-mode.

as shown in Fig. 4(b) and (c), respectively. The relation between the four-port and the two-port circuits are also indicated in the figure.

Fig. 5 and Fig. 6 present the extracted distributed $R L G C$ components for differential- and common-mode circuit models, respectively. As shown in Fig. 5(a) and Fig. 6(a), $R_{d d}$ and $R_{c c}$ increase with frequency due to skin effect and proximity effect. In addition, the observed $2 R_{c c}$ is higher than $R_{d d} / 2$, instead of equal in an ideal case. This can be attributed to the common-mode signal currents repel each other at the edges of the signal lines, while the differential-mode attract each other both due to proximity effect. Both effects reduce the current cross section but the repelled condition results in a relatively smaller area. The $L_{d d}$ and $L_{c c}$ decrease rapidly at low frequencies due to the current loop area is reduced both by skin and proximity effects. At high frequencies, the frequency

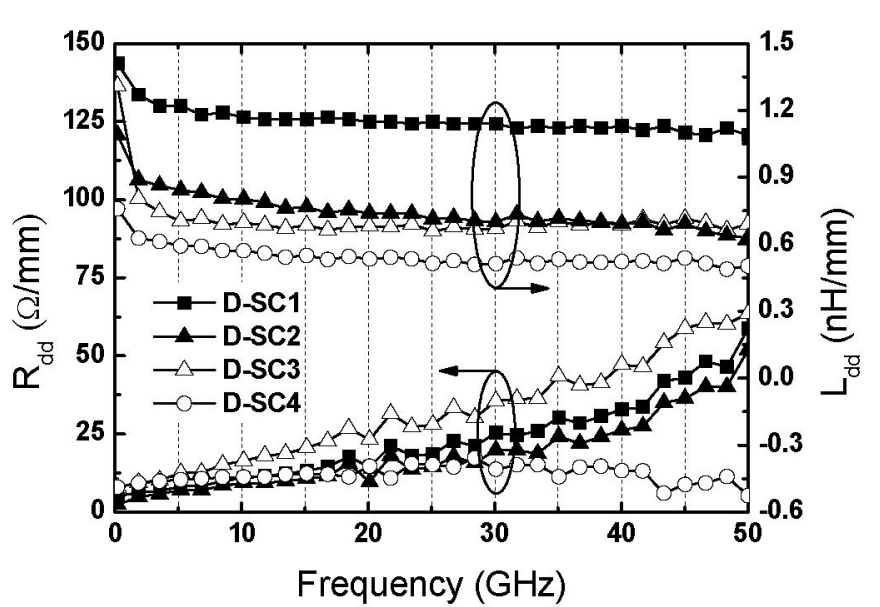

(a)

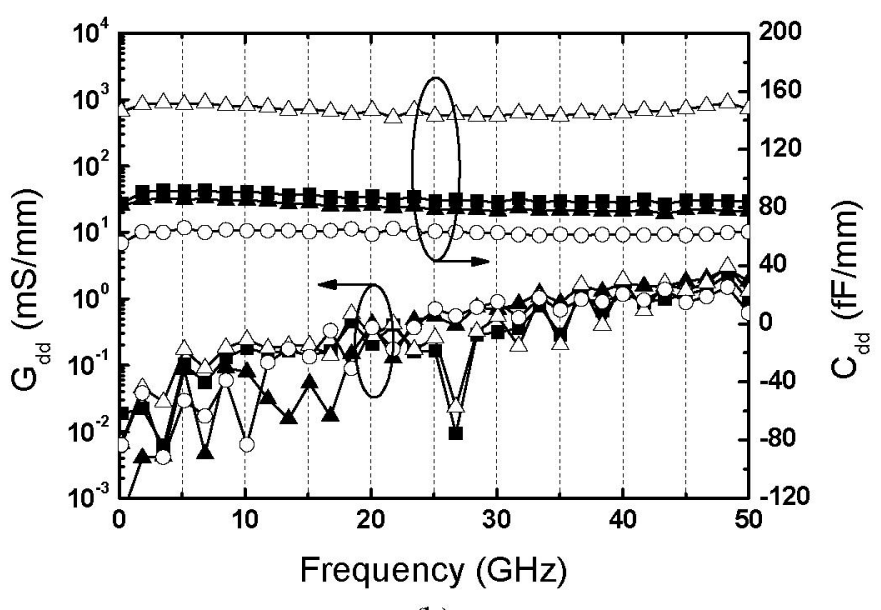

(b)

Fig. 5. Differential-mode (a) $R L$ and (b) $G C$ for D-SC lines.

dependence of inductance becomes smaller due to the total current loop area approaches a minimum value.

The increased $G_{d d}$ and $G_{c c}$ with frequency can be attributed to the dielectric conductivity, which is proportional to frequency, as shown in Fig. 5(b) and Fig. 6(b). In addition, the almost frequency-independent $C_{d d}$ and $C_{c c}$ suggest that the dispersion effect of the equivalent dielectric constant is very small.

\section{CONCLUSIONS}

In this paper, the low-loss S-SC and D-SC lines in a standard CMOS process were investigated for the first time. The S-SC and D-SC lines present the lowest $\alpha$ of $0.90 \mathrm{~dB} / \mathrm{mm}$ and $\alpha_{d d}$ of $\sim 1.00 \mathrm{~dB} / \mathrm{mm}$ at $50 \mathrm{GHz}$, respectively. The mixedmode S-parameters were adopted to characterize the four-port D-SC lines. The observed trends of distributed RLGC components of D-SC lines were explained in details. The results can be very useful for high-frequency interconnect 


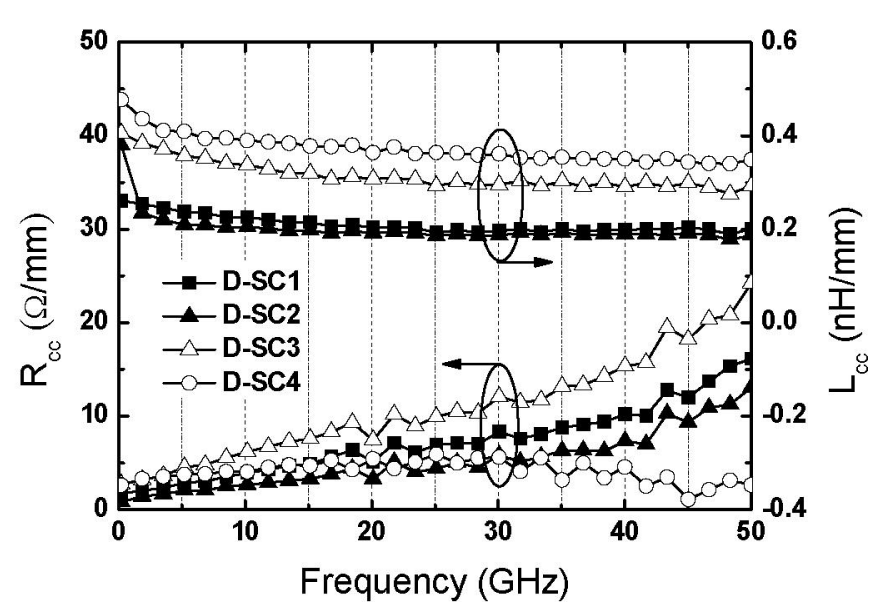

(a)

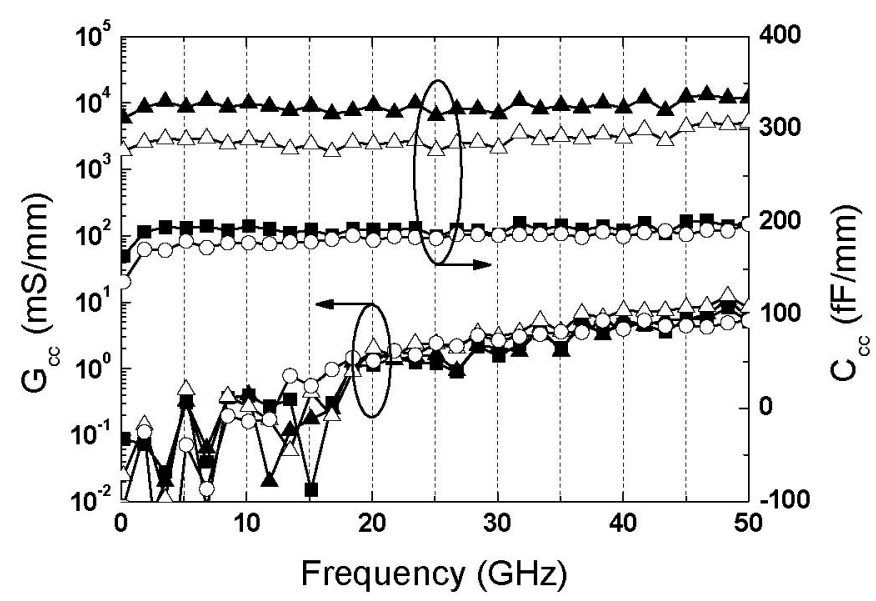

(b)

Fig. 6. Common-mode (a) $R L$ and (b) $G C$ for D-SC lines.

design optimization of both single-ended and differential circuits in standard CMOS processes.

\section{ACKNOWLEDGEMENT}

The authors would like to thank Miss S.-Y. Cho and Mr. Chih-Yuan Chan, and Chip Implementation Center (CIC) for the chip measurement. The authors would also like to express our gratitude for the financial support from NSC project (932215-E-007-023-) and the NTHU-TSMC Joint-Development Project.

\section{REFERENCES}

[1] R.-C. Liu, T.-P. Wang, L.-H. Lu, H. Wang, S.-H. Wang, and C.$\mathrm{P}$. Chao, "An $80 \mathrm{GHz}$ traveling-wave amplifier in a $90 \mathrm{~nm}$ CMOS technology," in IEEE Int. Solid-State Circuits Conf. Dig. Tech. Papers, pp. 154-155, Feb. 2005.

[2] P.-C. Huang, M.-D. Tsai, H. Wang, C.-H. Chen, and C.-S. Chang, "A $114 \mathrm{GHz}$ VCO in $0.13 \mu \mathrm{m}$ CMOS technology," in IEEE Int. Solid-State Circuits Conf. Dig. Tech. Papers, pp. 404405, Feb. 2005

[3] B. A. Floyd, S. K. Reynolds, U. R. Pfeiffer, T. Zwick, T. Beukema, and B. Gaucher, "SiGe bipolar transceiver circuits operating at $60 \mathrm{GHz}$," IEEE J. Solid-State Circuits, vol. 40, no. 1, pp. 156-167, Jan. 2005.

[4] M.T. Yang, P. P.C. Ho, T.J. Yeh, Y.J. Wang, D. C.W. Kuo, and et al., "On the millimeter-wave characteristics and model of onchip interconnect transmission lines up to $110 \mathrm{GHz}$," in IEEE MTT-S Int. Microwave Symp. Dig., pp. 1819-1822, June 2005.

[5] C. H. Doan, S. Emami, A. M. Niknejad, and R. W. Brodersen, "Millimeter-wave CMOS design," IEEE J. Solid-State Circuits, vol. 40, no. 1, pp. 144-155, Jan. 2005.

[6] B. Kleveland, T. H. Lee, and S. S. Wong, "50-GHz interconnect design in standard silicon technology," in IEEE MTT-S Int. Microwave Symp. Dig., vol. 3, pp. 1913-1916, June 1998.

[7] H. Ymeri, B. Nauwelaers, K. Maex, D. D. Roest, and S. Vandenberghe, "Accurate analytic expressions for frequencydependent inductance and resistance of single on-chip interconnects on conductive silicon substrate," Physics Letters $A$, vol. 28 , pp. 195-198, Jan. 2002.

[8] J. Kim, B. Jung, P. Cheung, and R. Harjani, "Novel CMOS lowloss transmission line structure," in IEEE Radio and Wireless Conf., pp. 235-238, Sept. 2004.

[9] D. E. Bockelman and W. R. Eisenstadt, "Combined differential and common-mode scattering parameters: theory and simulation," IEEE Trans. Microw. Theory Tech., vol. 43, no. 7, pp. 1530-1539, July 1995.

[10] G. Ghione and C. Naldi, "Parameters of coplanar waveguides with lower ground plane," Electronics Letters, vol. 19, no. 18, pp. 734-735, Sept. 1983.

[11] Y. Eo and W. R. Eisenstadt, "High-speed VLSI interconnect modeling based on S-parameter measurements," IEEE Trans. Comp., Hybrides, Manufact. Technol., vol. 16, no. 5, pp. $555-$ 562, Aug. 1993.

[12] S. Baek, S. Ahn, J. Park, J. Kim, J. Kim, and J.-H. Cho, "Accurate high frequency lossy model of differential signal line including mode-conversion and common-mode propagation effect," in Int. Symp. Electromagn. Compat., vol. 2, pp. 562-566, Aug. 2004. 